\title{
BMJ Open Population attributable fractions of modifiable cancer risk factors in Korea: a systematic review protocol
}

\author{
Mi Ah Han (D) , ${ }^{1}$ Eu Chang Hwang, ${ }^{2}$ Jae Hung Jung, ${ }^{3,4}$ Seo-Hee Kim, ${ }^{1,5}$ \\ Sun Mi Park ${ }^{1}$
}

To cite: Han MA, Hwang EC, Jung JH, et al. Population attributable fractions of modifiable cancer risk factors in Korea: a systematic review protocol. BMJ Open 2022;12:e055758. doi:10.1136/ bmjopen-2021-055758

- Prepublication history and additional supplemental material for this paper are available online. To view these files, please visit the journal online (http://dx.doi.org/10.1136/ bmjopen-2021-055758).

Received 22 July 2021 Accepted 05 January 2022

Check for updates

(C) Author(s) (or their employer(s)) 2022. Re-use permitted under CC BY-NC. No commercial re-use. See rights and permissions. Published by BMJ.

For numbered affiliations see end of article.

Correspondence to

Dr Mi Ah Han;

mahan@chosun.ac.kr

\section{ABSTRACT}

Introduction The burden of cancer continues to increase worldwide, and cancer is the leading cause of life expectancy reduction and death in Korea. Population attributable fraction (PAF), an epidemiological measure of exposures and health outcomes, could provide information on the public health impacts of exposures in populations. Knowing the PAFs of modifiable risk factors could aid in planning and prioritising strategies to reduce cancer burden in the population. This study aims to summarise systematically the PAF estimates of modifiable cancer risk factors in Korea.

Methods and analysis This review will include studies that determined PAFs of modifiable risk factors on cancer incidence and mortality in Korea. We will define modifiable risk factors as those that can be changed directly by peoples' conscious actions. We will perform systematic searches in EMBASE, MEDLINE, Cochrane Library and Korean databases (Korean Studies Information Service System, Research Information Sharing Service, KoreaMED, Korean Medical Database, National Assembly Library, and Korea Institute of Science and Technology Information) from their inception to July 2021. Two reviewers will independently screen studies for eligibility, extract data and perform quality assessments of the included studies. We will present the results in a qualitative or descriptive manner and will not perform meta-analyses or other quantitative data synthesis to derive summary estimates of PAFs because we anticipate high variability among PAF estimates.

Ethics and dissemination Ethics approval is not required because we will only use data from published papers. We will disseminate the results through publication in a peerreviewed journals.

PROSPERO registration number CRD42021268258.

\section{INTRODUCTION}

Cancer has been the primary cause of death in Korea since 1983 when causes of death were first reported. In 2019, cancer mortality was 158.2 per 1000000 , which accounted for $27.5 \%$ of total deaths. The most common causes of death were lung cancer, followed by liver, colorectal, gastric and pancreatic cancers. In 2018, there were 243837 new cancer cases in Korea and the most diagnosed cancers were stomach, followed by thyroid,

\section{Strengths and limitations of this study}

This systematic review will be the first to summarise population attributable fractions (PAFs) of modifiable cancer risk factors in Korea.

- We will use robust methods including a comprehensive search of eligible studies, explicit eligibility criteria, independent and duplicate eligibility screening, and data abstraction.

- Anticipated variability among PAFs may prevent a quantitative synthesis summary of the data.

lung, colorectal and breast cancers. The 5 -year cancer survival rate was $70.3 \%$ from 2014 to 2018, and total cancer cases were more than 2 million in $2018 .^{1}$

To reduce the growing cancer burden, the Korean government has implemented National Cancer Control Programmes. The government developed ' 10 codes for cancer prevention in Korea', which includes antismoking campaigns and a national vaccination programme targeting hepatitis B and human papillomavirus, as primary cancer preventions for the general public. In addition, a National Cancer Screening Programme for the early detection of cancer and a financial aid programme for cancer patients were developed. ${ }^{2}$

Population attributable fraction (PAF) is an epidemiological measure used widely to assess the public health impact of an exposure in a population. PAF is defined as the fraction of disease cases that could be avoided if the exposure were absent from the population. PAF measures the increased risk associated with the exposure as well as the prevalence of exposure. Therefore, PAF estimates can be used to plan and prioritise strategies to reduce cancer burden in the population.

When the exposure is modifiable, such as smoking, alcohol consumption and physical activity, the PAF can be interpreted as the preventable fraction of the disease, which can 
be prevented through removal of the exposure or elimination of risk factors. For example, the World Cancer Report 2008 reported that the PAF of tobacco use on cancer death was $32 \%$ in men, meaning that removing smoking may decrease cancer death $232 \% 00^{3}$ In addition, the $\mathrm{WHO} /$ International Agency for Research on Cancer announced that cancers could be reduced $\sim 30 \%-50 \%$ by removing modifiable risk factors. ${ }^{4}$

In 2009, the National Cancer Center Korea calculated PAFs of several modifiable risk factors, including tobacco use, ${ }^{5}$ chronic infection ${ }^{6}$ and alcohol consumption. ${ }^{7}$ Based on incidence, the most important risk factors for cancer were chronic infection, which contributed to $21.2 \%$ of new cancer cases and $24.7 \%$ of cancer deaths, ${ }^{6}$ followed by tobacco use, which contributed to $11.8 \%$ of new cancer cases and $22.7 \%$ of cancer deaths. ${ }^{5}$

The term 'attributable' has a causal implication, thus PAFs should be calculated using data from studies that adhere to rigorous methodologies. However, previous studies on PAFs of modifiable cancer risk factors did not consider the quality of the included studies and the data were not up-to-date. There is no systematic summary of PAF data on cancer in Korea. Therefore, the purpose of this study is to summarise systematically the PAF data on modifiable risk factors for cancer incidence and mortality in Korea.

\section{METHODS AND ANALYSIS}

We will follow the Preferred Reporting Items for Systematic Review and Meta-Analysis Protocols checklist in reporting the protocol. ${ }^{8}$

\section{Eligibility criteria}

The included studies will meet the following criteria:

1. Calculated PAF estimates for cancer incidence or mortality.

2. Focused on the Korean population.

3. Consideration of at least one of the modifiable risk factors.

4. Relative risks (RR) predominantly from meta-analyses of cohort studies or large cohort studies.

5. Prevalence data from nationally representative surveys, cohort studies or cross-sectional studies.

Modifiable risk factors are those in which people could change their exposure status directly through conscious action. Common examples include health behaviours (eg, smoking, alcohol consumption, physical activity, diet), preventive health service uptake (eg, vaccination) and environmental exposure (eg, herbicide use).

Non-modifiable risk factors are those that people cannot change or that are very difficult or unusual to change, such as age, gender, ethnicity, family history of disease and genetic characteristics (eg, mutation, gene expression). Biological status, such as blood pressure and blood lipids, can be modified through lifestyle changes or medication rather than by direct action; therefore, it will not be regarded as modifiable for this study.
We will include studies addressing overall cancer risk and specific cancer risk. A study that reported global or continent-level PAFs along with a subgroup analysis of Korea will be eligible for this study. A study that used indirect data, such as RRs, from Asian countries to calculate PAFs for Koreans will be eligible. However, if all of the data used to calculate PAFs, such as prevalence of exposure data and RRs, came from Asian countries outside of Korea, the study will be ineligible. If a study reported only the PAF of multiple risk factors such as health behaviour cluster, we will exclude the study since we are interested in the PAF of individual risk factors. There will be no language restriction. We will exclude studies in which prevalence of exposure or RRs were calculated from casecontrol studies. We will also exclude umbrella reviews and protocols that do not contain original data.

\section{Literature search}

We will conduct literature searches in EMBASE, MEDLINE, Cochrane Library and Korean databases, including Korean Studies Information Service System, Research Information Sharing Service, KoreaMED, Korean Medical Database, National Assembly Library and Korea Institute of Science and Technology Information from their inception to July 2021. We will develop a search strategy through consultation with an experienced librarian. Online supplemental table 1 presents the search strategy.

\section{Study selection}

Two reviewers will independently screen titles and abstracts for eligibility. Then, the reviewers will independently screen full-texts for potentially eligible studies. Discrepancies among the reviewers will be resolved through discussion or consultation with a third reviewer if needed.

\section{Data abstraction}

We will collect the following information from each eligible study:

1. Study information: first author, publication year, study design (cohort, other).

2. Population: population type (national, local), area (if local), population characteristics.

3. Modifiable risk factors: type of modifiable risk factor (eg, smoking, alcohol drinking), prevalence of exposure, source of exposure data (primary study, national health data, other), number of included participants, method of exposure assessment.

4. RR: type of outcome (incidence, mortality), type of cancer, type of RR (HR, other), source of RR (primary study, meta-analysis, other).

5. PAF: PAF estimate, calculation method, year of PAF estimate, latency period applied to PAF.

Two reviewers will extract the data independently and discrepancies will be resolved by discussion or consultation with a third reviewer if necessary. 


\section{Risk of bias}

Two reviewers will independently assess the risk of bias in the included studies and will resolve disagreements by consensus or discussion with a third reviewer. Risk of bias will be assessed using the Clarity group risk of bias tool for cohort studies. When RR comes from meta-analysis data, we will use A MeaSurement Tool to Assess systematic Reviews-2. ${ }^{9}$ Reviewers will rate each modifiable risk factor-cancer pair in each study and will resolve discrepancies by discussion or consultation with a third reviewer if needed.

\section{Analysis and summary of findings tables}

We will present descriptive statistics for the general characteristics of the included studies. We will not conduct meta-analysis or other quantitative synthesis to pool estimates because we anticipate variable PAF estimates. The best PAF estimates will be presented in a summary of findings table. If more than one PAF is reported for a modifiable exposure-cancer pair, we will select the best and most representative PAF based on the following: (a) if more than one PAF is reported for a modifiable exposure-cancer pair, we will choose the PAF calculated with Korean data only, (b) if PAFs at both the national and large cohort (city) levels are reported, we will choose the national level PAF, (c) if the PAF is based on a specific cohort study, we will select the PAF from the study with the larger sample size. We will present the selected PAF estimates to the summary of findings tables and present all other PAFs to online supplemental tables.

\section{Patients and public involvement}

No patients or public will be involved.

\section{DISCUSSION}

This will be the first systematic review of PAFs of modifiable cancer risk factors in Korea. The cancer burden has increased in Korea and is expected to continue to increase. This review will provide PAFs of various modifiable risk factors on cancer, and the findings could be used to prioritise and plan strategies to reduce cancer burden. The strength of this review is that it will use a systematic and transparent procedure with rigorous standards, including a comprehensive search of eligible studies, explicit eligibility criteria, independent and duplicate eligibility screening, data abstraction and risk of bias assessment. However, anticipated variability among PAFs may prevent a quantitative synthesis summary of the data.

\section{Ethics and dissemination}

This systematic review will not require approval from the institutional review board. We will disseminate the findings of this systematic review through peer-reviewed publication.

\section{Author affiliations}

${ }^{1}$ Department of Preventive Medicine, College of Medicine, Chosun University, Gwangju, Republic of Korea

${ }^{2}$ Department of Urology, Chonnam National University Medical School, Chonnam National University Hwasun Hospital, Hwasun, Republic of Korea

${ }^{3}$ Department of Urology, Yonsei University Wonju College of Medicine, Wonju, Republic of Korea

${ }^{4}$ Center of Evidence Based Medicine, Institute of Convergence Science, Yonsei University, Seoul, Republic of Korea

${ }^{5}$ Department of Public Health, Graduate School, Chosun University, Gwangju, Republic of Korea

Contributors MAH conceived the study. MAH, ECH and JHJ designed and developed the study design. MAH, SHK and SMP drafted the manuscript. All authors critically reviewed and finalised the protocol.

Funding This research is supported by the Basic Science Research Programme through the National Research Foundation of Korea (NRF) funded by the Ministry of Education (2021R111A3041301).

Competing interests None declared.

Patient consent for publication Not applicable.

Provenance and peer review Not commissioned; externally peer reviewed.

Supplemental material This content has been supplied by the author(s). It has not been vetted by BMJ Publishing Group Limited (BMJ) and may not have been peer-reviewed. Any opinions or recommendations discussed are solely those of the author(s) and are not endorsed by BMJ. BMJ disclaims all liability and responsibility arising from any reliance placed on the content. Where the content includes any translated material, BMJ does not warrant the accuracy and reliability of the translations (including but not limited to local regulations, clinical guidelines, terminology, drug names and drug dosages), and is not responsible for any error and/or omissions arising from translation and adaptation or otherwise.

Open access This is an open access article distributed in accordance with the Creative Commons Attribution Non Commercial (CC BY-NC 4.0) license, which permits others to distribute, remix, adapt, build upon this work non-commercially, and license their derivative works on different terms, provided the original work is properly cited, appropriate credit is given, any changes made indicated, and the use is non-commercial. See: http://creativecommons.org/licenses/by-nc/4.0/.

ORCID iD

Mi Ah Han http://orcid.org/0000-0003-1213-6952

\section{REFERENCES}

1 Hong S, Won Y-J, Lee JJ, et al. Cancer statistics in Korea: incidence, mortality, survival, and prevalence in 2018. Cancer Res Treat 2021;53:301-15.

2 National cancer control programs in Korea. J Korean Med Sci 2007;22(Suppl):S3-4.

3 Boyle P, Levin B. World cancer report 2008. IARC Press, International Agency for Research on Cancer, 2008.

4 World Health Organization. Cancer: World Health Organization, 2021. Available: https://www.who.int/health-topics/cancer [Accessed $16 \mathrm{Jul}$ 2021].

5 Park S, Jee SH, Shin H-R, et al. Attributable fraction of tobacco smoking on cancer using population-based nationwide cancer incidence and mortality data in Korea. BMC Cancer 2014;14:406.

6 Shin A, Park S, Shin HR, et al. Population attributable fraction of infection-related cancers in Korea. Ann Oncol 2011;22:1435-42.

7 Park S, Shin H-R, Lee B, et al. Attributable fraction of alcohol consumption on cancer using population-based nationwide cancer incidence and mortality data in the Republic of Korea. BMC Cancer 2014;14:420.

8 Moher D, Shamseer L, Clarke M, et al. Preferred reporting items for systematic review and meta-analysis protocols (PRISMA-P) 2015 statement. Syst Rev 2015;4:1.

9 Shea BJ, Reeves BC, Wells G, et al. AMSTAR 2: a critical appraisal tool for systematic reviews that include randomised or nonrandomised studies of healthcare interventions, or both. BMJ 2017;358:j4008. 